\title{
Complex Systems: Phenomenology, Modeling, Analysis
}

Aggelos C. Iliopoulos

Laboratory of Mechanics and Materials, Aristotle University of Thessaloniki, Thessaloniki 54124, Greece

\begin{abstract}
In this paper a short overview on complex systems and their basic features, as well as the models and mathematical tools developed for their analysis, is given. This review is formed according to the related experience, activity and scientific interests of the author, namely focused on mainly in nonlinear time series analysis and statistics and their applications on different physical systems. In particular, rough outlines are given concerning the phenomenology of complex systems, e.g. far from equilibrium thermodynamics and Tsallis statistics, power law scaling, multi-fractality, low dimensional chaos, SOC, strange kinetics and anomalous diffusion and turbulent intermittency. In addition, a non-complete list of models, based on equations or agent based, is briefly described such as Kuramoto - Sivashinsky equation, cubic complex Ginzburg-Landau Equation, reaction-diffusion Equation, fractional equations, cellular automata, complex networks and artificial neural networks. A more extended review is provided concerning the nonlinear time series analysis complex systems, describing tools like mutual information, flatness coefficient, structure functions, Tsallis q-triplet, correlation dimension and Lyapunov exponents in the reconstructed phase space, which can provide valuable information for the complex system's dynamics. Finally, applications of nonlinear time series analysis on various physical systems, such as earthquakes, Earth's magnetosphere, solar plasma and solar wind, plastic deformation of materials, epilepsy, economical indices and DNA structure, are provided.
\end{abstract}

\section{Complex Systems}

In general, even though there is no precise definition of complex systems, a complex system can be thought of as a collection of nonlinearly interacting elements summing up as a whole which is characterized by novel large scale effects. These effects arise as emergent properties related with nonlinear-complex behavior, which is the most distinguishing feature of complex systems [1]. A nonexhaustive list of complex systems contains among others (in fact most systems can be considered as complex systems): geophysical processes (earthquakes), biophysics (brain dynamics, DNA), space plasmas (solar wind, solar flares, Earth's magnetosphere), plastic deformation of materials, economy (stock indices), socio-technical systems and many others. The aforementioned complex systems, even though they are completely different in many aspects (e.g. different elements), share common characteristics such as:

1. They consist of a large number of interacting elements. This fact allows the description of these systems in two different hierarchical levels, namely microscopic and macroscopic.

2. Their subsystems and their interactions are nonlinear.

3. They exhibit emergent behavior, namely a self-organizing collective behavior, which is not predictable from the knowledge of the element's behavior.

4. These systems are open, namely they interact with their environment. This allows these systems to be driven to metastable states far from equilibrium, where self-organization and collective behavior manifest.

These systems are usually spatially distributed systems [2]. They are of infinite dimension, since in order to be completely determined would require an infinite number of initial conditions. They can be thought of as a synthesis of many dynamical subsystems, each of which has its own dynamics. Their dynamical behavior varies in space and time leading to the emergence of spatio-temporal patterns [3]. In addition, since they are open systems, they can be considered as driven systems, exhibiting input-output (or equivalently load-unload) and threshold dynamics. In such systems, nothing happens since an external source (driver) exceeds a specific value (threshold) [4]. However, since the system is driven far from equilibrium, its' behavior is characterized by fast unload processes in form of bursts, which produce avalanche events, giving rise to long range correlations in space and time. A representative paradigm of such a complex system is seismogenesis. In particular, earthquakes are caused by the deformation and sudden rupture of some parts of the Earth's lithopshere. In this case the driving force is convection in the mantle which drives the plate tectonic motions. Under appropriate conditions, the energy stored in the Earth's crust is released in a "bursting" mode, namely the earthquakes. Therefore, the interaction between the lithosphere and the mantle convection indicates the fact that plate tectonics and lithospheric internal dynamics, related with seismogenesis, constitute an input-output driven process [5].

This paper is summarized as follows: In chapter 2, the phenomenology and the basic characteristics of complex systems are presented, while in chapter 3 an overview of models used frequently in the study of complex systems is given. Chapter 4 is an introduction to extended nonlinear time series analysis presenting also some basic tools, while chapter 5 presents its application to some indicative complex systems. Finally, in chapter 6 a summary is given.

\section{Phenomenology of Complex Systems}

The phenomenology of the nonlinear behavior of complex systems includes emergent collective phenomena such as: far from equilibrium dynamics, power law scaling, multifractality, chaos, self organized criticality (SOC), intermittent turbulence, avalanches,

"Corresponding Author: Dr. Aggelos C. Iliopoulos, Laboratory of Mechanics and Materials, Aristotle University of Thessaloniki, Thessaloniki 54124, Greece, E-mail: ailiopou@gmail.com

Citation: Iliopoulos AC (2016) Complex Systems: Phenomenology, Modeling, Analysis. Int J Appl Exp Math 1: 105. doi: https://doi.org/10.15344/ijaem/2016/105

Copyright: (C) 2016 lliopoulos. This is an open-access article distributed under the terms of the Creative Commons Attribution License, which permits unrestricted use, distribution, and reproduction in any medium, provided the original author and source are credited. 
non-Gaussian statistics, anomalous diffusion etc [7], to name a few. In the following we will briefly describe the most significant of them:

\section{Input-Output, threshold dynamics}

In general, the dynamics of an input-output complex system can be described by the differential equation [6]

$$
\frac{\mathrm{d} \boldsymbol{X}}{\mathrm{dt}}=\boldsymbol{f}(\boldsymbol{X}(\mathrm{t}), \boldsymbol{U}(\mathrm{t}), \lambda)
$$

The internal state of the system is described by the vector $\boldsymbol{X}$ and the input $U$ by the vector, while $\lambda$ is the control parameter. For spatially distributed systems, $\boldsymbol{X}$ and $\boldsymbol{U}$ are fields $\boldsymbol{X}=\boldsymbol{X}(\boldsymbol{r}, \boldsymbol{t}), \boldsymbol{U}=\boldsymbol{U}(\boldsymbol{r}, \boldsymbol{t})$ and their dynamics can be described by partial differential equations

$$
\frac{\partial \boldsymbol{X}}{\partial \mathrm{t}}=\boldsymbol{f}\left(\{\boldsymbol{X}(\boldsymbol{r}, \mathrm{t}), \boldsymbol{U}(\boldsymbol{r}, \mathrm{t})\}, \nabla^{\mathrm{k}}\{\boldsymbol{X}(\boldsymbol{r}, \mathrm{t}), \boldsymbol{U}(\boldsymbol{r}, \mathrm{t}), \lambda(\boldsymbol{r}, \mathrm{t})\}\right)
$$

In addition, in an input-output process the internal dynamics of the system is coupled with its environment and the output is a signal(s) $z(t)$, which can be observed or measured. This signal includes valuable information for the internal dynamics as well as for the input dynamics according to the relation

$$
\mathrm{z}(\boldsymbol{r}, \mathrm{t})=\Phi(\boldsymbol{X}(\boldsymbol{r}, \mathrm{t}), \boldsymbol{U}(\boldsymbol{r}, \mathrm{t}))
$$

\section{Far from equilibrium thermodynamics}

The rich and interesting behavior of complex systems usually takes place in conditions far from equilibrium. In this paragraph we briefly describe the notion of entropy $S$ since it is the most significant notion concerning thermodynamics. We also introduce Tsallis entropy which is nowadays widely used in describing various complex systems' statistics.

It is well known that in isolated systems, the energy is conserved and entropy is monotonically increases till it reaches its maximum, a state which is known as thermodynamic equilibrium

$$
\frac{d S}{d t} \geq 0
$$

However, as we noted in the paragraph 2.1, most complex systems interact with their environment, namely they are open systems. In such systems, there exists a flow of entropy between the (sub)-systems of the system in study and its environment. In this case, the entropy change $\mathrm{dS}$ during a time interval $\mathrm{dt}$, can be decomposed into two components [8],

$\frac{d S}{d t}=\frac{d S_{i}}{d t}+\frac{d S_{e}}{d t}$

where $\frac{d S_{i}}{d t} \geq 0$ (the entropy produced inside the system can never be negative) is the entropy production due to the irreversible processes inside the system (e.g. diffusion, heat conduction, chemical reactions, others) and $\frac{d S_{e}}{d t}$ (it can attain negative values) is the entropy flux due to exchanges of energy or matter with the environment. As it can be seen, it is possible for the overall entropy to be negative in nonequilibrium conditions, a fact that is connected with the emergence of ordered patterns called dissipative structures (something that it cannot be observed in closed systems).

These far from equilibrium, macroscopic, large-scale order structures, correspond dynamically to non-equilibrium (quasi)stationary states (NESS's) [9], which have the topology of a percolating fractal set. The transition to a NESS is related to enhanced nonlinearities and developing of instabilities into a collective mode. In the NESS the system is in a turbulent state, exhibiting complex space distributions in the critical domain, while is fully determined by self organization processes which generate and maintain multi-scale correlations [9].

The most commonly used entropic function used for describing the thermodynamics of the complex systems is that based on BoltzmannGibbs (BG) statistics in which the entropy, for the discrete case, has the form of

$S_{B G}=-k \sum_{i=1}^{W} p_{i} \ln p_{i}$

where $W$ is a set of discrete states, $k$ is the Boltzmann constant $p_{i}$ is the probability of the (i) microscopic state of the system with

$$
\sum_{i=1}^{W} p_{i}=1
$$

However, even though BG statistics-thermodynamics is used efficiently to study many interesting systems and their behavior, it fails to describe ubiquitous phenomena commonly observed in complex systems and their basic quantities, since these quantities exhibit long range interactions, non-Gaussian and non-exponential behavior, for example power law scaling and multi-fractality. In order to confront with this problem, Tsallis [10] introduced a new definition of entropy, which is generalization of BG definition of entropy. In particular, this non-extensive entropy $\mathrm{Sq}$ is given by

$S_{q}=k \frac{1-\sum_{i=1}^{W} p_{i}^{q}}{q-1},\left(q \in \mathbb{R} ; S_{1}=S_{B G}\right)$

where $q$ is the entropic index, which is related to the microscopic dynamics and characterizes the degree of non-extensivity. In addition, it can be proved that $p_{i}^{q} \sim p_{i}\left[1+(q-1) \ln p_{i}\right]$.

For two independent systems $\mathrm{A}$ and B (e.g. $\left.p_{i j}^{A+B}=p_{i}^{A} p_{j}^{B}\right)$ then

$$
\frac{S_{q}(A+B)}{k}=\frac{S_{q}(A)}{k}+\frac{S_{q}(B)}{k}+(1-q) \frac{S_{q}(A)}{k} \frac{S_{q}(B)}{k}
$$

For all occasions $S_{q} \geq 0, q<1, q=1, q>1$ and the cases of correspond to super-additivity, additivity, sub-additivity, respectively.

As we will show in Chapter 5, a vast amount of papers nowadays concerning different complex systems showed that indeed Tsallis non-extensive statistical mechanics is appropriate for dynamical regions such as between regular motions and standard chaos, regions called edge of chaos, suggesting that the entropic index $q$ could be a convenient manner for quantifying some relevant aspects of complexity, such as (multi)fractal and similar, hierarchical, statistically scale-invariant, structures.

\section{Power law Scaling}

Power laws are considered to be a trademark of complex systems and appear in many cases of different complex systems, such as the ones mentioned in first paragraph. In general, a quantity $x$ obeys a power law if it derives from a probability distribution (PDF)

$$
p(x) \propto x^{-a}
$$

where a is a parameter of the PDF known as scaling exponent. If we plot Eq. 4 in log-log axes we will get a straight line, the slope of which is the value of a. This law is also known as Zipf's law or Pareto distribution [11]. Sometimes a power law distribution is also said to be a scale invariant distribution, because it remains the same under any scale transformation. 


\section{Multi-fractality}

Power laws are closely related to fractals [12]. Indeed, fractals are geometrical objects which cannot be described by Euclidean geometry since they possess a fractal dimension, they are characterized by selfsimilarity and they do not have a specific length. Paradigms are the trees, the brain, the clouds, the coast-shores and many others. For the characterization of fractal objects different fractal dimensions were developed such as the Hausdorff dimension $D_{H}$, the capacity dimension $D_{0}$, the information dimension $D_{1}$, the correlation dimension $D_{2}$ etc. In particular, an object is said to be fractal if parts or segments $N$ of the object scale as a power law with an exponent $D$ $N(L)=A L^{-D}$

where $\mathrm{A}$ is a constant and the exponent $\mathrm{D}$ fractal dimension.

However in many cases a simple fractal dimension cannot describe a complex inhomogeneous geometrical object. Then, this complexity can be described efficiently by the concept of multi-fractality [13]. In this case, the fractal distribution attains different degrees of clustering in different regions in the object. Therefore, multi-fractality quantifies the degree of clustering and the intermittency in a fractal object. For characterizing a multi-fractal object $S$ the generalized fractal dimension is estimated, from which the other fractal dimension are derived also

$$
D_{\bar{q}}(\bar{q})=\lim _{\varepsilon \rightarrow 0} \frac{I(\bar{q}, \varepsilon)}{\log \varepsilon}
$$

where the function $I(\bar{q}, \varepsilon)$ is given by

$$
I(\bar{q}, \varepsilon)=\frac{1}{1-\bar{q}} \log \sum_{i=1}^{N(\varepsilon)} P(i, \varepsilon)^{\bar{q}}
$$

where $P(i, \mathcal{E})^{\bar{p}}$ is the $\bar{q}_{\text {th }}$ power of the probability that points of the object $S$, are in the cell trying to "cover" the object $S$, with "spheres" of magnitude $\varepsilon$ and $\mathrm{N}(\varepsilon)$ is the number of the "spheres". For a monofractal object, $D_{0}=D_{1}=D_{2}=D_{3}=\ldots$, while for a multifractal object these dimensions are not the same.

\section{Low Dimensional Chaos}

As we mentioned before complex systems can exhibit rich behavior depending on the conditions and or/the control parameter. In particular, as, for example, a control parameter is increased and $t \rightarrow \infty$ the dynamical behavior of the system can be characterized in the phase space by limit cycles, low dimensional torus and low dimensional chaotic (strange) attractors. Low dimensional chaotic behavior was initially discovered on the behavior of a rather simple nonlinear system, described by three nonlinear differential equations, the well known Lorenz system [14]. In particular, low dimensional chaos describes the irregular-random behavior which emerges from a rigorous deterministic evolution of a low dimensional system in time, without the presence of any source of noise or external stochasticity. This aperiodic behavior manifests as sensitivity to initial conditions, namely nearby trajectories in the phase space diverge exponentially as time passes, excluding a long term prediction of the systems' dynamics. This phenomenon is also called "butterfly effect", is due to the nonlinear coupling between the variables of the system [15]. In the phase space, the strange attractor is a low dimensional confined set which is characterized by a fractal dimension, has self-similar properties and close trajectories diverge exponentially as time passes. Such a behavior can also be observed also in complex systems with many, practically infinite, degrees of freedom. Specific examples of such behavior are well studied in hydro-dynamics and refer to Rayleigh-Benard convection, Taylor-Couette flow and dripping faucet model [16-18].

\section{Self Organized Criticality (SOC)}

Another characteristic behavior of complex systems is the SOC behavior, which is strongly connected with power law scaling. This behavior is very different from the chaotic one we described in the previous paragraph, since in this case the system is high dimensional and is characterized by a zero maximum Lyapunov exponent. In particular, the theory of SOC by Bak, Tang kal Wiesenfeld [19] was developed in order to explain the power law behavior of various systems such as earthquakes, solar storms, snow avalanches and many others. According this theory, dissipative spatially distributed systems far from equilibrium can physically evolve to critical point states without any characteristic temporal or spatial scale. In these critical points, the states of the systems are characterized by power laws of temporal correlations (e.g. flickering $1 / \mathrm{f}$ noise) and spatial correlations namely spatial fractal structures. In addition, these points are attractors for the system, are not being affected from various parameters of system and are solely due to the nonlinear interactions of the elements of the system. That is why it is called self organized. The fluctuations around the SOC state are called avalanches and characterized by long range correlations. Very small fluctuations can lead to avalanches of all sizes. Practically, the SOC mechanism involves slow accumulation of energy and fast unloading of this energy through avalanches. Finally, according to [19], in a SOC system the divergence of near-by trajectories follow a power law, instead of an exponential law which is characteristic of chaotic systems. In addition, the degrees of freedom are proportional to the size of the system, namely the system is high dimensional (practically infinite), in contrast with the low dimensional chaotic systems. The SOC attractor corresponds to a kind of weak chaos (zero maximum Lyapunov exponent) and the systems lives at the edge of chaos.

\section{Strange Kinetics and Anomalous Diffusion (Transport) Processes}

Complex systems are often connected to strange kinetics which involve anomalous diffusion (transport) processes, both sub-linear and super-linear [20], giving rise to long range correlations which manifest as non-Gaussian ('strange') behavior of the system's kinetic behavior near non-equilibrium stationary states [9]. A common way to measure transport (diffusion) processes is to study the time evolution of the mean square displacement of the particle

$$
\left\langle\mathbf{r}^{2}(t)\right\rangle=2 \mathrm{D} \times t^{a}
$$

where $\mathrm{D}$ is the normalization constant having the meaning of the generalized transport coefficient and the exponent classifies the type of transport: for $a=1$, the diffusion is normal, for $a \neq 1$, anomalous. In particular, for $0<a<1$ there exists sub-diffusion and for $a=1$ superdiffusion [22,23]. Normal diffusion is usually related to systems near thermodynamical equilibrium, where the trajectories of the particles are irregular but homogeneous, namely they consist of small similar distances, whereas anomalous diffusion is connected with systems far from equilibrium, where the trajectories are very inhomogeneous. This inhomogeneity, is due to the fact that particles are "trapped" into eddies where they stay for unknown time and in the following they go through big "flights", meaning they travel long distances. In addition, anomalous diffusion can also be connected with power law distributions, called Levy distributions. In this case, the "flights" in anomalous diffusion processes are called Levy flights, the waiting times are described by Poissonian distributions, whereas the distribution of flights $\lambda(x)$ [23] are power law distributions according to the relation

$$
\lambda(x) \simeq|x|^{-1-\mu}(0<\mu<1)
$$


Intermittency

An additional complex dynamical behavior in spatio-temporal extended driven systems is intermittency in turbulence. In particular, in classical fluid turbulence, scaling is often associated with a hierarchical structure of eddies extending over the inertial range $[24,25]$. In this picture (called cascade model), the shear friction between these two distinct formations in the flow creates, in a little bit downstream, big eddies with the diameter about $l_{\text {in }}$. The eddies flow downstream with the mean current producing smaller eddies one after another. As a result, a turbulent state is formed comprised of a mixture of various sizes of eddies from large to small (fully developed turbulence). The diameter of the eddies is given by

$l_{n}=l_{i n} \delta_{n}, \delta_{n}=\delta^{-n}(n=0,1,2, \ldots), \quad \delta>1$

Here, $n$ is the number of steps in the cascade producing smaller eddies in a stream. Therefore, turbulence is fluctuating with different rhythms, intermittently, which allow one to observe the intermittent burst of fluctuations. The cascade model, described previously, was introduced to account for these intermittent phenomena. The inertial range is the region where the energy of eddies are delivered, consecutively, to smaller eddies and the diameter of eddies satisfy the relationship $l_{i n}>>l_{n}>>n$. The highly intermittent character of turbulent phenomena manifested in complex systems, is also connected with inhomogeneity in fluctuations in different quantities of the turbulent system. This inhomogeneity is manifested as small-scale fluctuations of high intensity surrounded by extended areas of much lower fluctuations. Therefore, intermittency manifests itself via the burst-like behavior in temporal and spatial domains. In addition, intermittency is strongly related to non-Gaussian statistics and multifractality (since a Gaussian process is mono-fractal). Indeed, in this cascade picture of turbulence many multifractal models were developed such as the lognormal model, $p$-model, Arimitsu and Arimitsu model [25].

\section{Modeling Complex Systems}

A nonlinear complex system can be studied in various ways, e.g either macroscopically studying its behavior, either mesoscopically studying the operation of its subsystems, either microscopically. Even though the study of complex systems requires a vast amount of information, the development of computers provided and provides great help in understanding complex systems. In this direction various theoretical and computational models were developed which can be classified into two great categories [1]:

\section{$\rightarrow$ Equation-Based \\ o Partial Differential and Differential Equations \\ o Maps}

$\rightarrow$ Agent Based, such as cellular automata and complex networks

Some representative examples of mean field type models are the Kuramoto - Sivashinsky equation [26], the complex GinzburgLandau equation [27], the reaction diffusion-equation [28], and others. In the following we describe briefly some partial differential equations commonly used to model complex systems' behavior.

\section{Kuramoto - Sivashinsky equations}

This equation is widely studied in various phenomena such as plasma physics, flows, convection, chemical reactions and others, exhibiting a variety of nonlinear and turbulent states, such as low dimensional chaos, found in spatially extended systems [26]. Its form can be written as

$$
\partial h / \partial t=-\nabla^{2} h-\nabla^{4} h+(\nabla h)^{2}
$$

where $h(\mathbf{X}+t)$ can be interpreted as the height of a d-dimensional interface embedded in $d+1$ dimensions.

\section{Cubic complex Ginzburg-Landau equations}

Another well studied nonlinear equation is the cubic complex Ginzburg-Landau equation which can efficiently describe a vast variety of nonequilibrium phenomena in spatially extended systems, from nonlinear waves to second-order phase transitions, from superconductivity, superfluidity, and Bose-Einstein condensation to liquid crystals and strings in field theory [27]. It has the form $\partial_{t} A=A+(1+i b) \Delta A-(1-i c)|A|^{2} A$

where $A$ is a complex function of (scaled) time $t$ and space $x$ and the real parameters $b$ and $c$ characterize linear and nonlinear dispersion.

\section{Reaction-Diffusion equations}

These equations are widely applicable in many areas, including chemistry, biology, physics and engineering [28]. The simplest reaction-diffusion equation can be written as

$$
\partial_{t} \mathbf{q}=\underline{D} \nabla^{2} \mathbf{q}+R(\mathbf{q})
$$

where $\underline{D}$ is the diffusion constant, and $\mathrm{R}$ is a nonlinear function representing the reaction kinetics. The nontrivial dynamics of these models arises from the competition between the reaction kinetics and diffusion and their solutions exhibit a rich behavior, similar to those found in non-equilibrium spatially distributed complex systems.

Fractals are closely related to complex systems. As we showed they are irregular over all length scales (non-integer dimensions) and this fact raises serious difficulties in studying them with usual calculus, which is unable in describing efficiently such structures and processes. Indeed, fractal functions do not possess first-derivative at any point. Therefore, new mathematical operators are needed and these can be found in fractional calculus [29]. In the last decade many fractional equations were developed, including diffusion equations, wave equations, relaxation equation (for a review see [30].

The most frequently used definition of a fractional integral of order $p(p>0)$ is the Reimann-Liouville's definition,

$$
\frac{\mathrm{d}^{p} f(x)}{[\mathrm{d}(x-a)]^{p}}=\frac{1}{\Gamma(n-p)} \frac{\mathrm{d}^{n}}{\mathrm{~d} x^{n}} \int_{a}^{x} \frac{f(y)}{(x-y)^{p-n+1}} \mathrm{~d} y
$$

with $n-1<q<n$ ( $n$ integer) as the $n$th integer of the $(n-q)$ th fractional integral. The above formula is a direct generalization of Cauchy's formula for repeated integration

$$
\frac{\mathrm{d}^{-p} f(x)}{[\mathrm{d}(x-a)]^{-p}}=\frac{1}{\Gamma(p)} \int_{a}^{x} \frac{f(y)}{(x-y)^{1-p}} \mathrm{~d} y
$$

When $p$ is not an integer, the fractional derivative (20) is a nonlocal operator since it depends on the lower integration limit $a$. In addition, when $a=0$, the following scaling property exists

$$
\frac{\mathrm{d}^{p} f(b x)}{[\mathrm{d} x]^{p}}=b^{p} \frac{\mathrm{d}^{p} f(b x)}{[\mathrm{d}(b x)]^{p}}
$$

providing a link between fractional calculus and fractals and $p$ being the fractal dimension [31], since the fractional operator shows the same scaling laws as the $a$-dimensional Hausdorff measure of a fractal set $V$, for e.g. $M_{a}(b V)=b^{a} M_{a}(V)$. 
Page 5 of 11

\section{Cellular automata}

One class of models used to describe complex systems and their behavior, without the use of differential equations is cellular automata (CA), which are examples of mathematical systems constructed from many identical components, each simple, but together capable of complex behavior [32,33]. A 1-D CA consists of a line of sites ("cells"), with each site carrying a value of $(0, \ldots, k-1)$. The state of the CA is completely specified by the values of the variables ai at each site $i$. The evolution of the CA takes place in discrete time steps and the variables are updated simultaneously based on the values of the variables in the neighborhood according to a deterministic rule

$a_{i}^{\{t+1\}}=\varphi\left[a_{i-r}^{t}, a_{i-r+1}^{t}, \ldots, a_{i+r}^{t}\right]$

The sites at microscopic level may represent points for example in a crystal lattice, while at macroscopic level a region of many molecules or others, depending on the scale and the system in study. In general, the overall behavior of CA can be extremely complex, since different rules yield different patterns that differ in detail but are similar in a statistical sense, exhibiting spatially homogeneous states, periodic structures, chaotic behavior, and complicated localized or propagating structures. The CA are used in modeling many physical complex systems such as earthquakes [19], brain dynamics [34] and many others.

\section{Complex networks}

Another promising tool for understanding complex systems and their behavior is complex network and network theory $[35,36]$. Examples of complex networks are systems composed by a large number of highly interconnected dynamical units, such as the Internet and the World Wide Web, neural networks, social interacting species, to name only a few. Complex networks can be studied either as graphs whose nodes represent the dynamical units, and whose links stand for the interactions between them, either as a dynamical system learning how a large ensemble of dynamical systems that interact through a complex wiring topology can behave collectively. Mathematically, a network is represented by a graph, consisting of a set of $\mathrm{N}$ nodes which are connected with a set of links. When a network is random, then the nodes are linked in a completely random manner. However, complex networks usually are far from random, often related to organizing principles. There are several measures to characterize network topology such as the clustering coefficient ci, the neighbor connectivity $\mathrm{knn}$, but the simplest is the degree distribution $\mathrm{p}(\mathrm{k})$, which is the probability for finding a node $i$ with degree $\mathrm{k}$ (number of links associated with it) in the network [37]. When the degree distribution is a power law, the network is called scale-free, which are of high interest nowadays, since, as we mentioned, many complex systems exhibit power law behavior, such as brain dynamics [38] and earthquakes [39].

\section{Artificial Neural Networks (ANNs)}

Very often complex systems are connected with a really vast amount of data, such as time series, images, various parameters, other spatiotemporal variables etc. For instance, for studying brain dynamics different variables are available such as interviews of patients and family members, physical exams, laboratory and cognitive tests and neurological exams, including magnetic resonance imaging (MRI) or computerized tomography (CT), positron emission tomography (PET) and functional magnetic resonance imaging (fMRI), as well as electroencephalography (EEG) time series. For the analysis and utilization of all this information advance techniques and algorithms are being developed summarized as machine learning. In particular, machine learning deals with algorithms that facilitate pattern recognition, classification and prediction, based on models derived from existing data [40]. Such computer algorithms included in machine learning arsenal are artificial neural networks (ANNs), which can recognize hidden patterns and relations of input data, model nonlinear complex functions, manage data and learn, improving their overall performance. Usually, ANNs consist of neurons or nodes which are grouped into layers: an input layer, one or several hidden layers and an output layer. The layers are connected with transfer functions and weights which are trained and initialized. A typical artificial neuron can be modeled as [41]: if we consider different inputs in a neuron, then the output signal $\mathrm{O}$ is given by:

$$
O=f(\text { net })=f\left(\sum_{j=1}^{n} w_{j} x_{j}\right)
$$

where $w_{j}$ is the weight vector, and the function $f(n e t)$ is referred to as an activation (transfer) function. The variable net is defined as a scalar product of the weight and input vectors

$$
\text { net }=w^{\mathrm{T}} x=w_{1} x_{1}+\ldots+w_{n} x_{n}
$$

where $T$ is the transpose of a matrix, and, in the simplest case, the output value $O$ is computed as

$$
O=f(\text { net })=\left\{\begin{array}{l}
1, w^{T} x \geq \theta \\
0, \text { otherwise }
\end{array}\right.
$$

where $\theta$ is called the threshold level; and this type of node is called a linear threshold unit. Paradigms of neural networks are [41] the radial basis functions (RBF) which use radial basis functions as activation functions, the probabilistic neural network (PNN) which uses a kernel-based approximation to for classification problems, the self organized maps which are used for dimensionality reduction of the data input. Applications of ANNs can be found in many scientific fields for e.g. the wind power prediction [42], in cancer prediction and prognosis [43], geomagnetic activity [44], earthquakes [45], etc

\section{Nonlinear Time series analysis and Statistics}

In this section we present a summary of algorithms and methods commonly used in the study of complex systems, based on nonlinear time series analysis. In particular, the main purpose of experimental time series analysis is to extract significant information for the underlying dynamics of the observed signal. Modern nonlinear time series analysis includes [46]:

i. Computation of Phenomenological Characteristics

(a) Autocorrelation Coefficient and Power Spectrum (Linear correlations, periodicities, scaling laws

(b) Mutual Information (Linear and Nonlinear Correlations)

(c) Probability Distributions (Power Laws)

(d) Hurst exponent (Persistence, anti-persistence, white noise)

(e) Flatness Coefficient F (Intermittent turbulence)

(f) Structure Functions (Turbulence, intermittency exponent)

(g) Phase portrait (Low Dimensionality)

(h) Entropy, energy, multifractal structures

(i) Estimation of q-Tsallis Statistics 
ii. Computation of Geometrical and Dynamical characteristics in the reconstructed state space

(a) Correlation Dimension (Degrees of freedom)

(b) Generalized Dimension (Multifractals)

(c) False Neighbors (Degrees of Freedom)

(d) Singular values spectrum (SVD components, filtering)

(e) Maximum Lyapunov Exponent (Sensitivity in initial conditions)

(f) Power Spectrum of Lyapunov Exponents

iii. Testing of Null Hypothesis in order to discriminate between low dimensional chaotic dynamics and linear high dimensional stochastic dynamics

(a) Surrogate data

(b) Discriminating statistics

iv. Singular Value Analysis in order to

(a) Estimate Degrees of Freedom

(b) Filter signals from White or Colored Noise

(c) Search for input-output dynamics

In the following we will present more analytically some of the most important tools of modern nonlinear time series analysis:

\section{The mutual information}

The mutual information can be used to search for nonlinear correlations between the values of an experimental signal. In general, the mutual information between two observables, A and B is given by the relation

$$
I_{A B}=H(A)-H(A / B)=H(A)+H(B)-H(A, B)
$$

where $H(A)$ is the amount of average information gained from a measurement of $A$ and $H(A / B)$ is the amount of information of $A$ given that $B$ is known. If this relation is applied to time series, it leads to [47]:

$$
\begin{aligned}
& I(\tau)=-\sum_{x(i)} P(x(i)) \log _{2} P(x(i))-\sum_{x(i-\tau)} P(x(i-\tau)) \log _{2} P(x(i-\tau))+ \\
& +\sum_{x(i)} \sum_{x(i-\tau)} P(x(i), x(i-\tau)) \log _{2} P(x(i), x(i-\tau))
\end{aligned}
$$

If the samples $\{B=x(i)\}$ and $\{A=x(i-\tau)\}$ are statistically independent then the mutual information will vanish for this value of $\tau$. Thus, knowledge of the second sample cannot be gained by knowing the first. On the other hand, if the first sample uniquely determines the second sample then $I(\tau)=I_{\text {max }}$, which is true when $\tau=0$. For a statistically independent signal, the mutual information is zero for $\tau>0$.

\section{Flatness coefficient $F$}

The intermittent nature of a complex system's dynamics can be investigated through the Probability Density Functions (PDF) of a set of two-point differenced time series of an original time series $\delta B_{\tau}(t)$ $=B(t+\tau)-B(t)$, which can be any physical quantity. The coefficient $\mathrm{F}$ corresponding to the flatness values of the two-point difference for the observed time series is defined as [48]:

$$
F=\frac{<\delta B_{\tau}(t)^{4}>}{<\delta B_{\tau}(t)^{2}>^{2}}
$$

The coefficient $\mathrm{F}$ for a Gaussian process is equal to 3, while deviation from this value imply non-Gaussian behavior and intermittency. The parameter $\tau$ represents the spatial size of the "eddies", which contribute to the energy cascade process.

\section{Structure functions}

Another way to search for intermittency, is the generalized structure functions, which are the various order moments of the fluctuating quantity, namely a time series,

$$
S_{p}(\tau)=\langle|s(t+\tau)-s(t)|\rangle^{p}
$$

where $\langle\ldots\rangle$ is the ensemble average of experimental time series. The structure functions should obey a power law as a function of lag time $\tau$

$$
S_{p}(\tau) \sim \tau^{J(p)}
$$

The scaling index as function of the moment $\mathrm{p}$, can be used to characterize the turbulent field [49], since deviation of $J(p)$ from $p / 3$ implies non-Gaussian behavior and intermittency.

\section{The Tsallis $q$-triplet estimation}

The most basic of Tsallis indices $q_{\text {sensitivity }}, q_{\text {relaxation }}, q_{\text {stationary }}$, known also as Tsallis q-triplet [50], constitute the best empirical quantifier of non-extensivity. In the following we describe briefly the underlying mathematical framework concerning Tsallis $q$-triplet:

\section{Tsallis index $q_{\text {sen }}$.}

This index is related to q-exponential sensitivity to initial conditions and to the rate of entropy production of the system in study. The $q_{\text {sen }}$ index is given by

$q_{\text {sen }}=1-\frac{a_{\text {max }} a_{\text {min }}}{a_{\text {max }}-a_{\text {min }}}$

The $a_{\max }$ and $a_{\min }$ values correspond to zeros of multifractal spectrum function, which is estimated by the Legendre transformation $f(a)=$ $\bar{q} a-(\bar{q}-1) D_{\bar{q}}$, where describes the Rényi generalized dimension of the solar wind time series according to

$D_{\bar{q}}=\frac{1}{q-1} \cdot \lim \left(\log \sum p_{i}^{q} / \log r\right)$ for $r \rightarrow 0$

\section{Tsallis Index $q_{\text {stat }}$}

The estimation of Tsallis q entropic index, referred to as stationary $q$ $=q_{\text {stat }}$, is related to the size of the distribution tail and is usually based on Tsallis $q$-Gaussian distributions which can describe metastable stationary states of the system. The Tsallis $q$-Gaussian distribution [51] is given by

$G_{q}(\beta ; x)=\frac{\sqrt{\beta}}{C_{q}} e_{q}^{-\beta x^{2}}$

where $\mathrm{e}_{\mathrm{q}}=[1+(1-q) x]^{1 /(1-q)}$ is the $q$-exponential, $\beta$ is a positive number and $C_{q}$ is a normalization constant, namely $C_{q}=\int_{-\infty}^{\infty} e_{q}^{-x^{2}} d x$. Depending on the $q$ value, $C_{q}$ has the following forms 


$$
\begin{aligned}
& C_{q}= \frac{2 \sqrt{\pi} \Gamma\left(\frac{1}{1-q}\right)}{(3-q) \sqrt{1-q} \Gamma\left(\frac{3-q}{2(1-q)}\right)},-\infty<q<1 \\
& C_{q}=\sqrt{\pi}, q=1 \\
& C_{q}=\frac{\sqrt{\pi} \Gamma\left(\frac{3-q}{2(q-1)}\right)}{\sqrt{q-1} \Gamma\left(\frac{1}{q-1}\right)}, 1<q<3
\end{aligned}
$$

For $q<1$, the support of $G_{q}(\beta ; x)$ is compact since this density vanishes for $|x|>1 / \sqrt{(1-q) \beta}$ q

\section{Tsallis Index $q_{\text {rel }}$}

This index is related to relaxation processes of the system. It is given by $q_{r e l}=(s-1) / s$, where $s$ is the slope of the log-log plotting of mutual information $I(\tau)$ given in paragraph 4.1 or the autocorrelation coefficient.

\section{The reconstructed state space}

Modern analysis of complex dynamical systems is also based on the reconstructed dynamics for autonomous and purely deterministic systems with $n$ dynamical variables, according to which a delay reconstruction map $\Phi$ is considered which maps the state $\boldsymbol{X}$ into the $m$ - dimensional delay vectors

$$
\Phi(\boldsymbol{x})=\left[h(\boldsymbol{x}), h\left(f^{\tau}(\boldsymbol{x}), \ldots, h\left(f^{(m-1) \tau}(\boldsymbol{x})\right)\right], \boldsymbol{x}(t) \in \mathbb{R}^{n}\right.
$$

This map is an embedding when $\mathrm{m} \geq 2 n+1$, where describes the dynamical flow underlying the observed signal and $n$ is the dimension of the manifold of the system phase space dynamics [52]. The embedding $\Phi$ is a diffeomorphism which maps the orbits of the original state space in the reconstructed space, preserving their orientation and dynamical and geometrical characteristics, such as Lyapunov exponents and dimension of attractors respectively. For the estimation of the best reconstruction time $\tau$, the first local minimum of the autocorrelation coefficient or the mutual information, as well as higher values of $\tau$ for which the estimated geometrical and dynamical characteristics of the reconstructed attractor remain invariable, can be used.

In the reconstructed state space we can also use the SVD (Singular Value Decomposition) analysis in order to: (i) filter the time series and (ii) decompose the series in its SVD reconstructed components which can be used for the detection of the underlying dynamics. Singular value analysis is applied to the trajectory matrix $X$ estimated by the reconstructed state space [53] The SVD analysis permits the reconstruction of the original flow, in terms of $n$ eigenvectors $V_{i}$, known as SVD reconstructed components corresponding to the spectrum of the singular values $\left\{\sigma_{\mathrm{i}}\right\}$. The $d$ largest ones of them correspond to the $V_{\mathrm{i}}$ eigenvectors, $i=1,2, \ldots \mathrm{d}$, that are sufficient for an accurate description of the underlying dynamics.

\section{Correlation dimension} by

The correlation dimension in the reconstructed phase space is given

$$
D_{m}=\lim _{r \rightarrow 0} \frac{d \ln C_{m}(r)}{d \ln (r)}
$$

of the dynamical trajectories of the system in state space, where $C_{m}(r)$ is the correlation integral of the trajectory and $D_{m}$ is its slope. The correlation integral is given by

$$
C_{m}(r)=\frac{2}{(N-W)(N-W-1)} \sum_{i=1}^{N} \sum_{j=1+W+1}^{N} \Theta(r-\|\mathbf{x}(i)-\mathbf{x}(j)\|)
$$

The low value saturation $D=\lim _{m \rightarrow \infty} D_{\mathrm{m}}$ of the slopes of the correlation integrals is related to the number $\mathrm{d}$ of fundamental coordinates of the internal dynamics. The method of Theiler [54] is also used to exclude $W$ time correlated states in the correlation integral estimation, thus discriminating between the dynamical character of the correlation integral scaling and the low value saturation of slopes characterizing self-affinity (or crinkliness) of trajectories in a Brownian process. When the dynamics possesses a finite (small) number of degrees of freedom, we observe saturation to low values $D$ of the slopes $D_{\mathrm{m}}$ obtained in (38) for a sufficiently large embedding $m$. The dimension of the attractor of the dynamics is then at least the smallest integer larger than D or at most, according to Takens' theorem.

\section{Lyapunov Exponents}

The Lyapunov spectrum is of high importance as it measures the rate of convergence or divergence of close trajectories in all $d$ directions of the phase space, giving further evidence for the possible low dimensional and chaotic character of the attractor in the reconstructed phase space. In particular, positive exponents correspond to instability, negative exponents to convergence, while at least one zero exponent must exist for the expansion along the trajectory. The Lyapunov spectrum is found by the derivative matrix of the dynamics in the reconstructed state space. The spectrum of Lyapunov exponents ordered as the $\lambda_{1} \geq \lambda_{1} \geq \ldots \geq \lambda_{d}$ by the relation [55].

$$
\lambda_{j}=\sum_{i=1}^{N} \log \left(\left\|\boldsymbol{A}_{i} \mathbf{e}_{j}^{i}\right\|\right)
$$

where $\boldsymbol{A}_{i}$ is the local approximation of the derivative matrix $D \mathbf{F}$ at the reconstructed trajectory points $\boldsymbol{x}_{\mathrm{r}}\left(t_{i}\right), i=1,2, \ldots, N$ of the $d-$ dimensional reconstructed state space and is a corresponding set of orthogonal vectors at the points $\boldsymbol{x}_{\mathrm{r}}\left(t_{i}\right)$.

\section{Surrogate data analysis}

The method of surrogate data is used to distinguish between linearity and nonlinearity as well as between chaoticity and pure stochasticity, since a linear stochastic signal can mimic a nonlinear chaotic process after a static nonlinear distortion, since they can mimic the geometrical or dynamical characteristics of the original data $[56,57]$. Therefore, they can be used for the rejection of every null hypothesis that identifies the observed low dimensional chaos as a purely non - chaotic stochastic linear process. In particular, surrogate data can be constructed according to [58], to mimic the original data, regarding their autocorrelation, power spectrum and their probability distribution.

In order to distinguish a nonlinear deterministic process from a linear stochastic one, we use as discriminating statistic a quantity $L$ derived from a method sensitive to nonlinearity, for example the correlation dimension, the maximum Lyapunov exponent, the mutual information etc. The discriminating statistic $L$ is then calculated for 
Page 8 of 11

the original and the surrogate data and the null hypothesis is verified or rejected depending on the "number of sigmas"

$$
\text { Sigma }=\text { Integer part }\left[\frac{i_{o b s}-i_{\text {sur }}}{o_{\text {sur }}}\right]
$$

where $\mu_{\text {sur }}$ and $\sigma_{\text {sur }}$ are the mean value and standard deviation of $L$ taken from the surrogate data and $\mu$ obs is the mean value of $L$ derived from the original data. For a single time series, $\mu$ obs is the single $L$ value [56]. The significance of the statistics is a dimensionless quantity and we report it in terms of units of Sigma "sigmas". When Sigma takes values higher than $2-3$ then the probability that the observed time series does not belong to the same family with its surrogate data is higher than $0.95-0.99$, correspondingly.

\section{Applications of Nonlinear Time Series Analysis in Various Complex Systems}

In the following we present recent representative studies (a nonexhaustive list, mostly the ones the author of this paper was involved with), concerning the application of nonlinear time series algorithms, described in previous paragraphs, in various physical systems, such as seismogenesis, plastic deformation of materials, space plasmas, brain dynamics, economy and DNA (see also [59]).

\section{Seismogenesis}

In a series of papers $[5,60-62]$ results were presented concerning seismogenesis in different Hellenic regions (land and sea of Greece), applying nonlinear analysis to various earthquake time series, such as magnitude, inter-event times, longitude and latitude time series. For the analysis, the model of the dripping faucet was used as a physical interpretation of the seismic process. In this model, the loading rate $\mathrm{m}(\mathrm{t})$ of mass in the mechanistic dripping faucet model of Shaw corresponds to the transfer of stress in the fault system by the mantle and plate tectonic dynamics (the external driver of the system), while mass unloading corresponds to earthquakes, as releases of the elastic strain energy stored along a fault. The dripping faucet similarly to the earthquake process can be understood as a local, driven, threshold process.

Geometrical and dynamical characteristics were then estimated in the reconstructed state space, such as the singular value spectrum, the correlation integrals and their slopes and the maximum Lyapunov exponent, for the original seismic time series and its SVD components. In addition, in order to exclude the case of linear stochastic dynamics that mimic low dimensional chaos, the authors compared their results with an efficient number of surrogate data. Tsallis statistics were also exploited to study the non-Gaussian character of the time series. Their results, showed that the spatio-temporal dynamics of earthquakes are of low dimensional, non-extensive, chaotic character, indicating that seismic events do not occur randomly in space and time and supported the hypothesis of seismogenesis, as an active chaotic walker, which corresponds to a point process realized in the continuously extended Hellenic lithospheric system. In addition, the nonlinear analysis of magnitude time series showed that an independent high dimensional SOC dynamics are connected with the energy release process. The above concepts showed that earthquakes can be understood via the general theory of statistical physics for dynamical processes of far from equilibrium phase transitions applied to distributed fault's systems.

\section{Space plasmas}

Space plasmas dynamics are also another example of complex distributed systems exhibiting complex behavior [63]. Studies concerning the nonlinear analysis of various time series derived from Earth's magnetosphere, solar wind, solar flares and sunspots others revealed the complex behavior of those systems, which include among others chaotic, SOC and intermittent turbulent dynamics, non-equilibrium phase transitions, as well as Tsallis non-Gaussian statistics [53, 64-71].

In particular, Earth's magnetosphere is strongly coupled externally to the solar wind plasma flow revealing dissipative internal nonequilibrium and non-linear dynamics, related with the development of magnetospheric superstorms during which strong plasma flows can be developed along the magnetotail. For example, in $[64,68]$, the analysis of $\mathrm{AE}$ index time series and bulk plasma velocity measurements (Vx component) of both calm and storm periods, revealed that during the development of a superstorm event, there is a phase transition of magnetopsheric dynamics from high dimensional (SOC state) to low dimensional and chaotic (chaos state), with a corresponding strengthening of the intermittent turbulent non-Gaussian character of the dynamics. The corresponding statistics can be well described within the theoretical framework of Tsallis statistics.

Similarly, the solar plasma dynamics is a prototype of non-linearity and non-integrability, exhibiting non-Gaussian turbulent and chaotic dynamics as well as spatial multifractal topology of the solar magnetic field. Solar activity is related to two different processes, corresponding to different regions of the solar system with different physical characteristics, namely sunspots in the photosphere and solar flares at the base of solar corona. The analysis of sunspot index and daily solar flares index time series $[65-67,69]$ revealed the coexistence of two clearly discriminated physical processes underlying the solar activity, corresponding to SOC and Chaos processes, related to photospheric and sub-photospheric zones activity of the Solar system. Also, strong evidence for intermittent solar turbulence as well as for non-extensive statistical processes, according to Tsallis q-statistics, was found.

Another example of complex system is solar wind plasma. It consists of ionized and magnetized gas, composed mainly by protons, electrons, alpha particles and heavier ions, continuously flowing away from the solar corona in all directions pervading the interplanetary space. Its dynamics is also a nonlinear, far from thermodynamical equilibrium in which the development of hierarchical, self-organized and long-correlated dynamical states is possible. Indeed in recent studies [70-71] the non-extensive and non-Gaussian character of the solar wind plasma was verified, indicating the existence of multi-scale strong correlations from the microscopic to the macroscopic scales. These long range correlations are significantly enhanced during the phase transition from calm to "shock" events, as the analysis of Tsallis q-triplet and other nonlinear quantities revealed.

\section{Unstable plastic flow in deformation of materials}

Plasticity in material's deformation is a highly complex spatiotemporal phenomenon. The complexity of the underlying dynamics is mainly connected to complex geometric objects, dislocations, which not only act as carriers of deformation, but they move, interact and evolve in a nonlinear way, resulting in a nonuniform and non-isotropic, non-random spatial distribution [72]. One representative paradigm of such an unstable plastic flow is the 
Page 9 of 11

Portevin-Le Chatelier (PLC) effect, which is manifested when some dilute alloys undergo tensile tests in specific regimes of strain rate and/ or temperature and is connected with serrations in stress/strain graphs and shear bands in material's surface. Recently, [73] conducted Tsallis statistical analysis for various stress serration time series of $\mathrm{Cu}-15 \% \mathrm{Al}$ alloys corresponding to different deformation temperatures and PLC types (A, B, C), estimating Tsallis' entropic index $q \equiv q_{\text {stat }}$ (stat denotes stationary states). The results revealed the non-Gaussian (Tsallis q-Gaussian), nonextensive, sub-additive character of the underlying dynamics of the PLC type-A bands $(q>1)$ indicating dynamics at the edge of chaos, with global long range correlations and power law scaling. Similarly, for PLC type B bands, a Tsallis q-Gaussian, nonextensive, super-additive statistical profile was found with $q<1$, while for the PLC type C bands, a stochastic, near Boltzmann-Gibbs (BG) statistical character was verified indicating Gaussian dynamics with short (weak) range correlations. These results verify and extend previous studies [e.g.74] which classified type B and type A PLC bands underlying dynamics to distinct dynamical behavior, namely chaotic behavior for the first and self organized critical (SOC) behavior for the latter.

Complex behavior is also manifested in ultra fine grained (UFG) materials, in which the deformation mechanisms, involve grain rotation and grain boundary sliding, dislocation nucleation, emission and absorption in GBs, twinning and faulting as well as diffusional creep and grain boundary migration [75]. In a recent study [76], statistical features of serrations, which are observed in low strain rates, as well as the spatial distribution of extensive shear bands which are formed in high strain rates of an UFG alloys, were studied. The analysis was based on Tsallis nonextensive statistics and fractal theory. The results demonstrated that plastic flow that the statistics of serrations and shear bands' formation are connected with Tsallis q-Gaussians (q>1) and fractality [Iliopoulos et al., 2015b].

\section{Brain dynamics, Economics, DNA}

The human brain can also be modeled as a driven nonlinear threshold system, comprised of nonlinear units or cells, generating spatial complex networks, where each cell operates when the electrical potential or current reaches a threshold value. In [34] a phase transition process of brain activity was revealed from a high dimensional SOC state during the health period to a gradually developed low dimensional chaotic state corresponding to epileptic state. In addition, a cellular Automata (CA) model was developed which simulated faithfully the brain behavior during healthy and epileptic states.

Economics is also a very complex system. Indeed, various economic systems can exhibit ubiquitous complex dynamics evidenced by large amplitude and aperiodic fluctuations in economic and financial variables, including foreign exchange rates, gross domestic product, interest rates, production, stock market prices and unemployment. The presence of fluctuations in economic and financial systems are indications that these systems are driven far away from the equilibrium where non-linearity gives rise to complex system's behavior [77,78]. For example, the statistical analysis of two time stock market time series, namely Standard \& Poor's 500 (S \& P) 500 and TVIX [77], based on Tsallis non-extensive statistics and in particular the estimation of $q$ - triplet, showed the non-Gaussian, non-extensive statistical character of the time series, indicating multi-fractal phase space dynamics, which can be described faithfully by Tsallis distribution functions. The non-extensive character of the underlying dynamics is related to the existence of multi-scale long range interactions in space and time. In addition, the detailed analysis of S \& $P$ index unraveled the existence of non-equilibrium phase transitions depicted clearly in the variations of Tsallis $q$-triplet values, which are connected with non-equilibrium stationary states of economical dynamics derived from processes of strong self organization.

DNA is another example of complex system, since it can be thought of as a growth or aggregation phenomenon, which results in a fractal cluster with power-law correlations over wide ranges of length scales, resulting in long-range correlations of nucleotides in DNA sequences. In a recent study [79] applied an extensive nonlinear algorithm in DNA sequences of Major Histocompatibility Complex (MHC) showing that the DNA complexity and self-organization can be related to fractional dynamical nonlinear processes with low dimensional deterministic chaotic and non-extensive statistical character.

\section{Summary and Conclusions}

Complex systems and their behavior are found in many fields, such as scientific, engineering and social. These systems are much more than a sum of their parts and they are connected to the emergence of collective behavior through self-organization, e.g. the spontaneous formation of temporal, spatial or functional structures. They are also nonlinear, inhomogeneous, and far from equilibrium as well as adaptive as they evolve and may contain self-driving feedback loops. The collective behavior of the whole system cannot be simply inferred from the understanding of the behavior of the individual components [80].

Interesting features commonly emerged in complex systems such as far from equilibrium thermodynamics and Tsallis statistics, power law scaling, multi-fractality, low dimensional chaos, SOC, strange kinetics and anomalous diffusion and turbulent intermittency. For describing the complex behavior of such systems, new modeling tools are generated. A non-exhaustive list includes models based either on equations, for example Kuramoto - Sivashinsky equation, cubic complex Ginzburg-Landau Equation, reaction-diffusion Equation, fractional equations etc, or agent based such as cellular automata, complex networks, artificial neural networks (for further reading see also [81]). In addition, for the analysis of experimental data (time series) of complex systems new concepts and sophisticated mathematical tools are developed, roundup as nonlinear time series analysis, in order to extract significant information for the underlying dynamics of the observed signals. Tools like mutual information, flatness coefficient, structure functions, Tsallis q-triplet, correlation dimension and Lyapunov exponents in the reconstructed phase space can provide valuable information for the system's long range correlations, intermittent turbulence features, non-Gaussian statistics, degrees of freedoms and sensitivity to initial conditions.

As we presented in this paper, there is experimental evidence of intriguingly similar quantitative features traced from nonlinear time series analysis of data derived from different complex systems, such as earthquakes, Earth's magnetosphere, solar plasma and solar wind, plastic deformation of materials, epilepsy, economical indices and DNA structure. These results strongly support the concept of universality, namely that systems arising in different disciplines can share common characteristics and behavior, irrespective of the underlying microscopic mechanisms responsible for the observed complex behavior [82]. However, much remains to be explored. Various theoretical concepts and mathematical tools, among others, are now in intensive active research regarding complex systems, based on non-extensive statistical mechanics, fractal topology, turbulence 
theory, strange dynamics, percolation theory, anomalous diffusion theory and anomalous transport theory, fractional dynamics and non-equilibrium phase transition theory [ 83 and refs therein]. The advances in these theories will provide new insights in the description, analysis and understanding in the complexity of physical, engineering and social systems, leading to uncertainty and unpredictability reduction, which are fundamental aspects of these systems.

\section{Competing Interests}

The authors declare that they have no competing interests.

\section{Acknowledgments}

The author gratefully acknowledges Prof. G.P. Pavlos, Dr. M. Athanasiou, Dr. L.P. Karakatsanis, E. Pavlos and M. Xenakis for various useful discussions on complexity theory and complex systems.

\section{References}

1. Ninno B (2004) Modelling Complex Systems, New York Inc : Springer.

2. Cai D, McLaughllin DW, Shatah J (2001) Spatiotemporal Chaos in Spatially Extended System, IMACS 55: 329-340.

3. Addison Paul S (1997) Fractals and Chaos, IOP.

4. Rundle JB, Klein W, Gross S, Ferguson CD (1997) Traveling density wave models for earthquakes and driven threshold systems. Phys Rev E 56: 293- 307.

5. Pavlos GP, lliopoulos AC, Athanasiou M (2007) Self Organized Criticality or / and Low Dimensional Chaos in Earthquake Processes. Theory and Practice in Hellenic Region. Eds. Tsonis A. and Elsner J. Nonlinear Dynamics in Geosciences, Springer, pp. 235-259.

6. Abarbanel HD, Brown R, Sidorowich JJ, Tsirming LS (1993) The analysis of observed chaotic data in physical systems. Rev Mod Phys 65: 1331-1392.

7. Pavlos GP, Iliopoulos AC, Tsoutsouras VG, Karakatsanis LP, Pavlos EG (2010) Spatiotemporal Chaos in Distributed Systems: Theory and Practice, in Chaotic Systems: Theory and Applications, Eds. Skiadas C.H. and Dimotikalis I., pp. 268-284, World Scientific.

8. Nicolis G (1977) Self Organization in nonequilibrium systems, John Wiley, 1977.

9. Zelenyi LM, Milovanov AV (2004) Physics-Uspekhi 47: 749-788.

10. Tsallis C (2009) Introduction to Nonextensive Statistical Mechanics, Springer.

11. Newman MEJ (2003) Power Laws, Pareto Distributions and Zipf's Law. Contemporary Physics 46: 323-351.

12. Mandlebrot BB (1983) The fractal geometry of nature. New York: Freeman.

13. Mandelbrot BB (1989) Multifractal Measures, Especially for the Geophysicist. PAGEOPH 131: 6-42.

14. Lorenz EN (1963) Deterministic nonperiodic flow. Journal of the Atmospheric Sciences $20: 130-141$

15. Ivancevic VG, Ivancevic TT (2007) High Dimensional Chaotic and Attractor Systems, Ed. Tzafestas S.G., Springer.

16. Yanagita T, Kaneko K (1995) Rayleigh-Benard convection, patterns, chaos, spatiotemporal chaos and turbulence, Physica D 82: 288-313.

17. Buzug Th, Stamm J, Pfister G (1992) Fractal Dimensions of strange attractors obtained from the Taylor-Couette experiment. Physica A 191: 559- 563.

18. Neda Z, Bako B, Rees E (1996) The dripping faucet revisited CHAOS 6: 59-62.

19. Bak P, Tang C, Wiesenfeld K (1987) Self-Organized Criticality: An Explanation of 1/f Noise, Phys. Rev. Lett 59: 381-384.

20. Shlesinger MF, Zaslavsky GM, Klafter J (1993) Strange Kinetics, Nature 363.

21. Schwammle V, Curadoa EMF, Nobre, FD (2009) Dynamics of normal and anomalous diffusion in nonlinear Fokker-Planck equations. Eur Phys J B 70: 107-116.

22. Rezende GR, Lapas LC, Oliveira FA (2009) Complexity perspectives: an anomalous diffusion approach, Journal of Computational Interdisciplinary Sciences 1: 105-111.
23. Metzler R, Chechkin AV, Klafter J (2009) Levy statistics and Anomalous Transports: Levy flights and Subdiffusion, Encyclopedia of Complexity and Systems Science, Ed. E. Meyers and A. Robert, 5218-5239, Springer.

24. Kolmogorov A (1941) Dokl. Akad. Nauk SSSR 31, 538.

25. Arimitsu T, Arimitsu N (2005) Multifractal analysis of the fat-tail PDFs observed in fully developed turbulence. Journal of Physics: Conference Series 7: 101-120.

26. Pandit R (1993) Universal Properties of the Two-Dimensional KuramotoSivashinky Equation. Phys Rev Lett 71: 12-15.

27. Aranson IS, Kramer L (2002) The world of the complex Ginzburg-Landau equation, Review of Modern Physics 74: 99-143.

28. Vastano JA, Russo T, Swinney HL (1990) Bifurcation to spatially induced chaos in a reaction-diffusion system. Physica D 46: 23-42.

29. Carpinteri A, Mainardi $F$ (1997) Fractals and fractional calculus in continuum mechanics, Wien: Springer.

30. Mainardi F (1996) Fractional relaxation-oscillation and fractional diffusionwave phenomena, Chaos, Solitons and Fractals 7: 1461-1477.

31. Carpinteri A, Cornetti P, Kolwankar KM (2004) Calculation of the tensile and flexural strength of disordered materials using fractional calculus, Chaos, Solitons and Fractals 21: 623-632.

32. Wolfram S (1983) Statistical mechanics of cellular automata. Rev Mod Phys 55: 601-644.

33. S Wolfram (1984) Cellular automata as models of complexity. Nature 311: 419-424.

34. Tsoutsouras V, Sirakoulis G, Pavlos G, lliopoulos A (2012) Simulation of Healthy and Epileptic form Brain Activity using Cellular Automata. International Journal of Bifurcation and Chaos 22: 1250229.

35. Newman MEJ (2003) The structure and Function of Complex Networks. SIAM Review 45: 167-256.

36. Boccalettia S, Latora S, Moreno Y, Chavez M, Hwang DU (2006) Complex networks: Structure and dynamics. Phys Rep 424: 175-308.

37. S Thurner (2005) Nonextensive statistical mechanics and complex scalefree networks, Europhysics News 36: 218-220.

38. K Spiliotis, Siettos C (2011) A timestepper-based approach for the coarse grained analysis of microscopic neuronal simulators on networks: Bifurcation and rare-events micro- to macro-computations, Neurocomputing 74: 3576-3589.

39. Daskalaki E, Papadopoulos GA, Spiliotis K, Siettos C (2014) Analysing the topology of seismicity in the Hellenic arc using complex networks. Journal of Seismology 18: 37-46.

40. Tarca AL, Carey VJ, Chen X, Romero R, Draghici S (2007) Machine Learning and Its Applications to Biology. PLoS Computational Biology 3: 953-963.

41. Abraham A (2005) Artificial Neural Networks in Handbook of Measuring System Design, Wiley.

42. Sideratos G, Hatziargyriou ND (2007) An advanced statistical method for wind power forecasting. IEEE Transactions on Power Systems 22: .

43. Cruz JA, Wishart DS (2006) Applications of Machine Learning in Cancer Prediction and Prognosis. Cancer Inform 2: 59-77.

44. Voros Z, Jankovicova D (2002) Neural network prediction of geomagnetic activity: a method using local Holder exponents, Nonlinear Processes in Geophysics 9: 425-433.

45. Reyes J, Morales-Esteban A, Martínez-Álvarez F (2013) Neural networks to predict earthquakes in Chile, Applied Soft Computing 13: 1314-1328.

46. Pavlos GP, lliopoulos AC, Karakatsanis LP, Xenakis M, Pavlos E (2015) Complexity of Economical Systems, Journal of Engineering Science and Technology Review 8: 41- 55.

47. Fraser AM, Swinney HL (1986) Independent coordinates for strange attractors from mutual information, Phys Rev A 33: 1134-1140.

48. Chapman SC, Hnat B, Rowlands G, Watkins NW (2005) Scaling collapse and structure functions: identifying self-affinity in finite length time series. Nonlinear Processes in Geophysics 12: 767-774.

49. Abramenko VI (2002) Solar MHD turbulence in regions with various levels of flare activity. Astron Rep 46 : 161-171.

50. Tsallis C (2004) Dynamical scenario for nonextensive statistical mechanics. Physica A 340: 1-10.

51. Umarov S, Tsallis C, Steinberg S (2008) On a q-Central Limit Theorem Consistent with Nonextensive Statistical Mechanics. MilanJMath 76: 307328. 
52. Takens F (1981) Detecting strange attractors in turbulence. Lectures Notes in Mathematics 898: 366-381.

53. Pavlos GP, Athanasiu MA, Diamantidis D, Rigas AG, Sarris ET (1999) Comments and new results about the magnetospheric chaos hypothesis. Nonlin Proces Geophys 6: 99-127.

54. Theiler J (1991) Some comments on the correlations dimensions of $1 / \mathrm{fa}$ noise. Phys Lett A 155: 480-493.

55. Kantz H, Schreiber T (1997) Nonlinear Time Series Analysis (Cambridge University Press).

56. Theiler J, LuDanK S, Longtin A, Galdrikian B (1992a] Using surrogate data to detect nonlinearity in time series", in Nonlinear Modeling and Forecasting vol. XII of SFI studies in the Sciences of Complexity, eds. Casdagli M. and Eubank S. (Addison-Wesley, Reading, Mass) pp. 163-188.

57. Theiler J, LuDanK S, Longtin A, Galdrikian B (1992b) Testing for nonlinearity in time series: the method of surrogate data. Physica D 58: 77-94.

58. Schreiber T, Schmitz A (1996) Improved surrogate data for nonlinearity test. Phys Rev Lett 77: 635-638.

59. Pavlos GP, Karakatsanis LP, Xenakis MN, Pavlos EG, lliopoulos AC, et al. (2014) Universality of Tsallis Non - Extensive Statistics and Time Series Analysis: Theory and Applications: Physica A 395: 58-95.

60. Iliopoulos AC, Pavlos GP, Athanasiu MA (2008) Spatiotemporal Chaos into the Hellenic Seismogenesis: Evidence for a Global Strange Attractor, NPCS 11: 274-279.

61. Iliopoulos AC, Pavlos GP (2010) Global Low Dimensional Seismic Chaos in the Hellenic Region. IJBC 20: 2071-2095.

62. Iliopoulos AC, Pavlos GP, Papadimitriou EE, Sfiris DS, Athanasiou MA, et al. (2012) Self Organized Criticality, intermittent turbulence and non-extensivity revealed from seismogenesis in North Aegean area. International Journal of Bifurcation and Chaos 22: 1250224

63. Pavlos GP, lliopoulos AC, Athanasiou MA, Karakatsanis LP, Tsoutsouras VG (2011a) Complexity in Space Plasmas: Universality of Non-equilibrium Physical Processes, in Modern Challenges in Nonlinear Plasma Physics, Ed. D. Vassiliadis, AIP Conf. Proc., Volume 1320: 77-81.

64. Pavlos GP, lliopoulos AC, Tsoutsouras VG, Sarafopoulos DV, Pavlos EG, et al. (2011b) First and Second Order Non-equilibrium Phase Transition and Evidence for Non-extensive Tsallis Statistics in Earth's Magnetosphere. Physica A 390: 2819-2839.

65. LP Karakatsanis, GP Pavlos (2008) Self organized criticality and chaos into the solar activity, Nonlinear Phenom. Complex Syst 11: 280-284.

66. Karakatsanis LP, Pavlos GP, lliopoulos AC, Tsoutsouras VG (2011) Evidence for Coexistence of SOC and Chaos processes in the Solar Flare Dynamics", in Modern Challenges in Nonlinear Plasma Physics, Ed. D. Vassiliadis, AIP Conf. Proc., Volume 1320: 55-64.

67. Pavlos GP, Karakatsanis LP, Xenakis MN (2012a) Tsallis non-extensive statistics, intermittent turbulence, $\mathrm{SOC}$ and chaos in the solar plasma. Part one: sunspot dynamics, Physica A 391: 6287-6319.

68. Pavlos GP, Karakatsanis LP, Xenakis MN, Sarafopoulos D, Pavlos EG (2012b) Tsallis statistics and magnetospheric self-organization. Physica $A$ 391: 3069-3080.

69. Karakatsanis LP, Pavlos GP, Xenakis MN (2013) Tsallis nonextensive statistics, intermittence turbulence, SOC and chaos in the solar plasma. Part two: solar flare dynamics, Physica A 392: 3920-3944.

70. Pavlos GP, lliopoulos AC, Zastenker GN, Zelenyi LM, Karakatsanis M, et al (2015) Tsallis Non-extensive Statistics and Solar Wind Plasma Complexity. Physica A 422: 113-135.

71. Patsourakos S, Georgoulis MK, Vourlidas A, Nindos A, Sarris T (2015) The major geoeffective solar eruptions of 7 March 2012: Comprehensive Sun to Earth Analysis, in press to The Astrophysical Journal.,

72. Aifantis EC (1987) The physics of plastic deformation, International Journal of Plasticity 3: 211-247.

73. Iliopoulos AC, Nikolaidis NS (2015a) Aifantis E.C Portevin Le Chatelier Effect and Tsallis Non-extensive Statistics. Physica A 438: 509-518.

74. Ananthakrishna G (2007) Current theoretical approaches to collective behavior of dislocations Physics Reports 440: 113-259.

75. Ovid'ko IA (2016) Aifantis EC. Rev Adv Mater Sci 35: 1-24.

76. Iliopoulos AC, Nikolaidis NS, Aifantis EC (2015b) Analysis of Serrations and Shear Bands Fractality in UFGs. Journal of the Mechanical Behavior of Materials 24: 1-9,.

77. Iliopoulos AC, Pavlos GP, Magafas L, Karakatsanis L, Xenakis M, et al. (2015c) Tsallis q-triplet and Stock Market Indices: The cases of S \& P 500 and TVIX. Jestr 8: 34-40.
78. Pavlos GP, lliopoulos AC, Karakatsanis L, Xenakis M, Pavlos E (2015c) Complexity of Economical Systems. Jestr 8: 41-55.

79. Pavlos GP, Karakatsanis LP, lliopoulos AC, Pavlos EG, Xenakis MN, et al. (2015d) Measuring Complexity, Nonextensivity and Chaos in the DNA Sequence of the Major Histocompatibility Complex, Physica A 438: 188209.

80. Meyers RA (2012) Mathematics of Complexity and Dynamical Systems, Springer.

81. CR Shalizi (2006) Methods and Techniques of Complex Systems Science: An Overview, in Complex Systems Science in Biomedicine, Eds. T.S. Deisboeck and J.Y. Kresh, Springer, 33-114.

82. Stanley HE, Amaral LAN, Gopikrishnana P, Ivanova Pch, Keittb TH, et al. (2011) Scale invariance and universality: organizing principles in complex systems, Physica A 281: 60-68.

83. Pavlos GP, lliopoulos AC, Karakatsanis LP, Tsoutsouras VG, Pavlos EG (2011) Complexity Theory and Physical Unification: From Microscopic to Macroscopic level, Chaos Theory: Modeling, Simulation, and Applications, (Eds.) Skiadas C.H., Dimotikalis I. and Skiadas C., pp. 297-308, World Scientific Publishing Co, 2011. 